\title{
Les interférences des écoles de pensée antiques dans la littérature de la Renaissance, sous la direction d'Edward Tilson
}

\section{Michele Mastroianni}

\section{(2) OpenEdition}

\section{Journals}

\section{Edizione digitale}

URL: http://journals.openedition.org/studifrancesi/1103

DOI: 10.4000/studifrancesi. 1103

ISSN: 2421-5856

\section{Editore}

Rosenberg \& Sellier

\section{Edizione cartacea}

Data di pubblicazione: 1 novembre 2014

Paginazione: 576

ISSN: 0039-2944

\section{Notizia bibliografica digitale}

Michele Mastroianni, «Les interférences des écoles de pensée antiques dans la littérature de la Renaissance, sous la direction d'Edward Tilson », Studi Francesi [Online], 174 (LVIII | III) | 2014, online dal 01 novembre 2014, consultato il 18 septembre 2020. URL : http://journals.openedition.org/ studifrancesi/1103; DOI : https://doi.org/10.4000/studifrancesi.1103

\section{Questo documento è stato generato automaticamente il 18 settembre 2020.}

\section{cc) $(9)$}

Studi Francesi è distribuita con Licenza Creative Commons Attribuzione - Non commerciale - Non opere derivate 4.0 Internazionale. 


\title{
Les interférences des écoles de pensée antiques dans la littérature de la Renaissance, sous la direction d'Edward Tilson
}

\author{
Michele Mastroianni
}

\section{NOTIZIA}

Les interférences des écoles de pensée antiques dans la littérature de la Renaissance, sous la direction d'Edward TiLson, Paris, Classiques Garnier («Études et essais sur la Renaissance», 100), 2013, pp. 242.

E. Tilson, organizzatore di questa interessante miscellanea, sottolinea giustamente nella sua prefazione come sia ormai lontana l'epoca in cui si studiavano i rapporti fra i testi rinascimentali e i loro modelli classici quasi si trattasse essenzialmente di un problema di fonti, e come ora si presti «une plus grande attention aux conditions qui président à la transmission des traditions philosophiques de l'Antiquité, à la chronologie de la reconstitution humaniste des corpus bien sûr, mais aussi à l'impact des sélections et des juxtapositions éditoriales comme des paratextes et des gloses qui les présentent». In questa prospettiva, i saggi contenuti nel volume esaminano le modalità con cui le assimilazioni e trasformazioni tra le scuole maggiori dell'epoca ellenistica, e i mutui scambi tra le correnti antiche, non soltanto producono interferenze con il pensiero rinascimentale, ma agiscono sulle forme e su generi letterari più disparati del Cinquecento. Riassumendo con il curatore, «l'essentiel de la pratique qu'illustre ce volume consiste en l'effort de considérer la fonction des éléments d'une école de pensée donnée non pas séparément ou dans leur rapport intertextuel avec le restant qu'un processus de soustraction aurait isolé comme le 
propre du texte, mais dans leurs interactions avec des éléments venus d'autres écoles et formes de pensée».

2 I contributi qui raccolti sono i seguenti: Violaine GIACOMOTTO-CHARRA, L'«Univers» de Pontus de Tyard à la croisée des chemins. Un exemple d'interférence scientifiques (pp. 21-51); Bénédicte Boudou, Henri Estienne et les interférences des écoles de pensée antique. Ou la défense de la vérité dans l'«Apologia pro Herodoto» (pp. 53-67); Dominique BRANCHER, De la suspension du Chyle. Sorbière en médecin sceptique (pp. 69-89); Sébastien PRAT, L'antilogie sceptique entre le Portique et la pénitence chrétienne dans le chapitre "du repentir» (pp. 93-111); Alain LEGROS, Sur bois et sur papier, les citations croisées de Montaigne (pp. 113-128); Edward TILSON, «Nous ne nous pouvons arracher de la vie par discours». La conférence de la théologie, de l'épicurisme et du scepticisme dans l'«Apologie de Raimond Sebond» (pp. 129-156); Anne-Pascale POUEY-MOUNOU, L'interface et l'intervalle. Le jeu des références savantes chez Ronsard et Muret (1552-1553) (pp. 159-180); Bernd RENNER, Satire et cynisme chez Juvénal et Martial. Aspects stylistiques de la satire renaissante (pp. 181-201); E. Bruce HAYES, Les perplexités de la masculinité. Cynisme, scepticisme et 'caritas' chrétienne dans le «Tiers Livre» de Rabelais (pp. 205-220); Nicola PANICHI, Le plaisir de la vertu entre étique et esthétique. Valla et Montaigne (pp. 221-234).

In una prima sezione («Les savoirs professionnels», pp. 21-89) si riflette sulle mutazioni che si verificano nei meccanismi di apprendimento legati a specifiche competenze: così, per esempio, V. Giacomotto-Charra evidenzia una modernizzazione degli studi filosofici - dell'aristotelismo, in questo caso - che passa attraverso il confronto di esercizi di scrittura differenti intorno a uno stesso oggetto, in un'indagine sull'effetto di specularità tra filosofia 'professionale' classica, filosofia umanistica e filosofia letteraria; così, B. Boudou, studiando la svolta umanistica della storiografia, analizza l'utilizzo da parte di Henri Estienne di scuole di pensiero classiche - riscoperte nel Cinquecento, come il pirronismo - in parallelo e in una continua interferenza con testi patristici nuovamente riattualizzati. In una seconda sezione («L'héritage chrétien dans les Essais», pp. 93-156) è il testo di Montaigne al centro di una riflessione sulla conférence dell'umanesimo volgare e dell'eredità cristiana. S. Prat sottolinea la centralità del pensiero stoico, analizzandone la centralità che esso occupa negli Essais (III, 2: «Du repentir») e dimostrando come sia necessaria una valutazione preliminare della struttura antilogica che governa la discussione a prima vista sconnessa del capitolo di Montaigne per comprendere il ruolo che vi esercitano i riferimenti a tradizioni diverse, anzitutto a quelle dello stoicismo. Così pure il problema del rapporto fra l'eredità cristiana e i lasciti filosofici dell'Antichità è affrontato dagli studi di A. Legros e E. Tilson sul tissage e métissage delle tradizioni classiche e cristiane, nella ricerca dei punti di intersezione, sempre in Montaigne, di tre tradizioni (quella biblica, quella di Lucrezio, quella scettica), in particolare nella ricerca di come le interferenze operino nell'apologia di Raimond Sebond. In una terza sezione («Les transformations poétiques», pp. 159-205) è la poesia ad essere il campo di indagine per individuare queste interferenze fra antico e moderno. Se A.-P. Pouey-Mounou esamina i rapporti fra poesia e filosofia in Ronsard, analizzando gli scarti fra le Amours e la loro ricezione nei commenti di Muret, B. Renner studia i rapporti tra la satira cinica classica, quale prende forma in Marziale e Giovenale, e la satira francese dal Rinascimento al Barocco, con alcune osservazioni che spaziano da Marot a Théophile de Viau. Infine, una quarta sezione («Repenser le plaisir», pp. 205-234) rivolgendosi al Rabelais del Tiers Livre e di 
nuovo a Montaigne affronta, nel quadro delle interferenze in questione, il problema della compatibilità tra l'amore cristiano, la caritas, e il piacere umano. 